\title{
WANDERING SPLEEN AS A CAUSE OF SINISTRAL PORTAL HYPERTENSION
}

\author{
Rodrigo Piltcher-da-Silva ${ }^{1}$, Vicente Lobato Costa ${ }^{1}$, Caroline Losekann ${ }^{1}$, \\ Luiz Roberto Rigo Wendt ${ }^{1}$, Eduardo Neubarth Trindade ${ }^{2}$
}

\begin{abstract}
Wandering spleen (WS) is a rare entity characterized by laxity of peritoneal ligaments that hold the spleen stationary. It is most commonly diagnosed in children and young women. Clinical presentation ranges from asymptomatic to acute abdomen. A 19-yearold woman came to the emergency department with history of progressive abdominal pain. She also had previous episodes of hematemesis. A computed tomography scan showed an ectopic spleen with a "whirlpool sign." Laparotomy and splenectomy were performed. WS is characterized by a long vascular pedicle and laxity of peritoneal attachments of the spleen. The etiology is usually congenital. Splenopexy is the main treatment; however, splenectomy is indicated when splenic infarction is present. Despite being rare, this condition may be considered in some cases of abdominal pain. An earlier diagnosis would have allowed us to perform a splenopexy, thus reducing morbidity.
\end{abstract}

Keywords: Case report; Spleen; Wandering spleen; Segmental portal hypertension; Acute abdomen

\section{INTRODUCTION}

Wandering spleen (WS), also called ectopic spleen, is a rare medical condition caused by laxity or absence of the splenic ligaments with congenital or acquired etiology ${ }^{1-4}$. It leads to migration of this organ from its anatomical location. Due to this dysfunction, the spleen can "wander" in the lower abdomen or pelvis and be mistaken as a pelvic mass. In case of delayed treatment, there may occur splenic pedicle torsion, infarction or necrosis ${ }^{1}$.

Only about 500 cases of WS have been reported in the literature ${ }^{5,6}$. The condition had an incidence lower than $0.25 \%$ in patients who required splenectomies, being more commonly diagnosed in young children or in women of childbearing age ${ }^{1,5,7}$. So far, WS is rarely considered when patients complain of abdominal pain and may be misdiagnosed if no complementary investigation is done.

The aim of this report is to share our experience with this rare case of WS with consequent development of sinistral portal hypertension in a 19-year-old female patient who was admitted to the emergency department (ED) with acute abdominal syndrome.

\section{PRESENTATION OF THE CASE}

A 19-year-old woman was referred from a hospital 56 miles away due to suspected splenic torsion. She had a 3-day history of progressive abdominal pain. A computed tomography (CT) scan performed at the hospital of origin had shown the spleen in pelvic position associated with a "whirlpool sign" suggesting pedicle torsion (Figure 1). Laboratory investigation at that time showed hemoglobin level of $12.4 \mathrm{~g} / \mathrm{dL}$ and no other abnormalities. The patient was given, initially, volume replacement and analgesia.
Clin Biomed Res. 2021;41(2):185-187

1 General Surgery Department, Hospital de Clínicas de Porto Alegre. Porto Alegre, RS, Brasil.

2 Digestive Surgery Department, Hospital de Clínicas de Porto Alegre. Porto Alegre, RS, Brasil.

Corresponding author: Rodrigo Piltcher-da-Silva rodrigopiltcher@gmail.com Hospital de Clínicas de Porto Alegre Av. Ramiro Barcelos, 2350 90035-903, Porto Alegre, RS, Brasil. 
Figure 1: Multislice CT scan images of abdomen. A: Coronal image shows that the spleen has migrated from the left hypochondrium and is ectopically located in the pelvic region; $\mathrm{B}$ : Axial image shows the twisted spleen pedicle; Blue arrow: spleen and red arrow - pedicle.
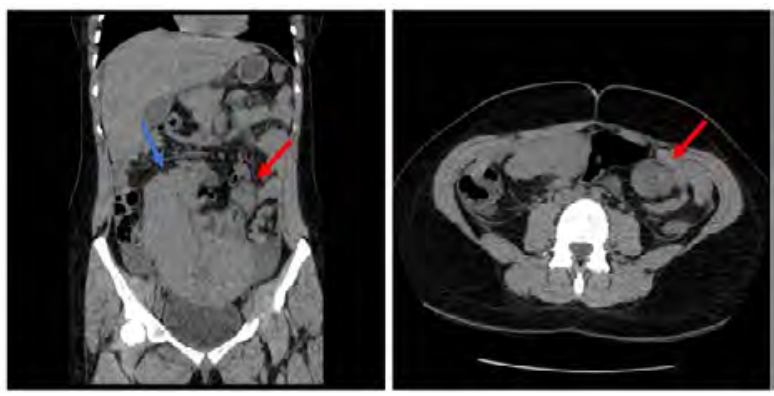

At admission, the patient had severe abdominal pain, signs of peritoneal irritation and a pulse rate of 128 beats/min, without any other abnormalities. Laboratory tests revealed hemoglobin level of $6.7 \mathrm{~g} /$ $\mathrm{dL}$ and creatinine level of $0.55 \mathrm{mg} / \mathrm{dL}$.

The patient was taken to the operating room to undergo an exploratory laparotomy. During surgery, the spleen was immediately identified in pelvic position with enlarged dimensions, clear signs of ischemia and a large pedicle with loops and thrombosis (Figure 2). Therefore, the surgery team opted for splenectomy instead of repositioning the spleen. There was no significant bleeding during surgery and the spleen was removed with no laceration (Figure 3). Pathological evaluation revealed hemorrhagic infarct of the spleen. The patient had a fine recovery and was discharged from hospital after 3 days.

Figure 2: Intraoperative findings. A: The spleen at an ectopic position on the pelvic region; $B$ : The spleen out of the abdomen cavity and its vascular pedicle with torsion (marked by a red arrow).
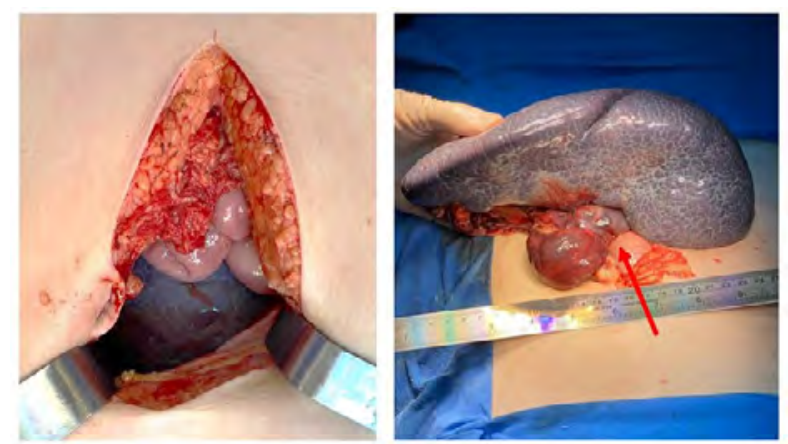

Further investigation showed that the patient had a past medical history of two upper gastrointestinal bleeding episodes with hemodynamic instability. Prior investigation of her condition in another hospital showed the presence of gastric varices at esophagogastroduodenoscopy with no evidence of hepatic disease, thus supporting the hypothesis of sinistral portal hypertension.

Figure 3: The surgical specimen with approximately $23 \mathrm{~cm}$ and large vascular pedicle.

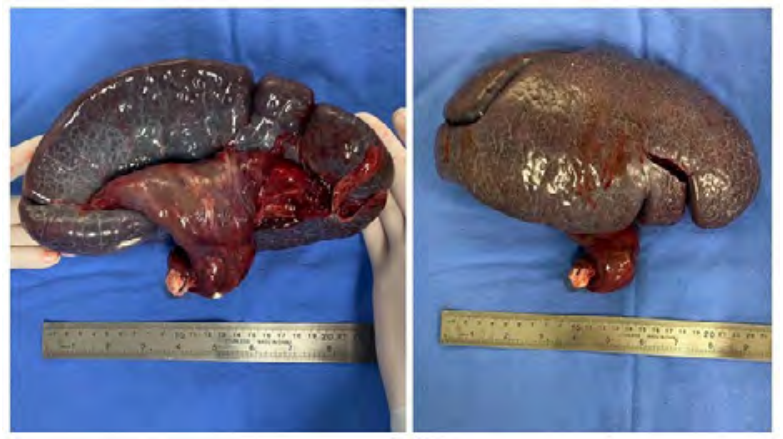

\section{DISCUSSION}

Wandering spleen is an extremely rare condition characterized by increased mobility of the spleen. It occurs due to a dysfunctional peritoneal attachment of its suspensory ligaments (gastrosplenic and splenorenal) ${ }^{8,9}$. The congenital etiology involves anomalies in the development of the dorsal mesogastrium during the second month of embryonic development and then failure of the dorsal mesogastrium to fuse into the posterior abdominal wall $1,8,10,11$. The consequence is an abnormally long splenic pedicle plus the absence or malformation of its ligaments 5 .

There are many factors involved in the acquired etiology, such as previous pregnancy, when the direct effects of estrogen will provoke laxity of those ligaments ${ }^{1,6}$. The condition also appears to be associated with Gaucher disease, absence of kidney, splenomegaly, malaria, Hodgkin disease and infectious mononucleosis ${ }^{1}$.

The clinical presentation of the condition ranges from completely asymptomatic, with incidental diagnosis during routine medical examination or imaging investigation, to acute abdominal syndrome ${ }^{12}$. When symptomatic, the most common sign is an abdominal or pelvic mass, which can be associated with gastrointestinal complaints such as nausea, emesis, cramps, and abdominal pain or even intestinal obstruction ${ }^{1,2}$. Furthermore, pedicle torsion and detorsion may cause recurrent pain. Acute abdominal syndrome in consequence of splenic pedicle torsion followed by infarction is the main presentation at the emergency department ${ }^{5,6,13}$.

Sinistral portal hypertension with fundal varices has been described in few reports and is related to splenic traction of gastroepiploic vein and absence of short gastric vessels ${ }^{9,14}$. Mesenteric varices are also a rare complication that can occur due to vascular traction ${ }^{9,15}$. Both fundal varices and mesenteric varices can be 
managed by splenectomy or splenopexy ${ }^{9}$. Laboratory tests are nonspecific and may reveal hypersplenism or functional asplenia. Howell-Jolly bodies will appear through peripheral smear in patients with asplenia1. Doppler sonography may show an ectopic spleen and is useful for identifying the vascular flow ${ }^{3}$. However, a CT scan with intravenous contrast is considered the best option to investigate the condition and may show the position and viability of the spleen ${ }^{3,6,12,16}$. It also shows the characteristic sign of a "whirled appearance" of the splenic pedicle ${ }^{2,9}$.

The best treatment in this situation is to perform a splenopexy even if the patient has acute abdominal pain as well as if pedicle detorsion is necessary ${ }^{3}$. However, this procedure can only be adopted if there is no evidence of infarction, thrombosis or hypersplenism ${ }^{1,16,17}$. Organ preservation is an important concern in young patients as they are at a high risk of developing an overwhelming post-splenectomy sepsis ${ }^{1,2,6,8}$. Splenectomy was once considered the first treatment option ${ }^{1}$, but nowadays it should only be performed in patients who have a contraindication for splenopexy ${ }^{3,9,12,18}$. Splenectomy is most frequently performed as an emergency procedure as described above. Therefore, the use of combined vaccines against Haemophilus influenzae, Streptococcus pneumoniae and Neisseria meningitidis is necessary for those patients who underwent splenectomy $y^{1,2,6}$.

WS is reported as a rare condition but must be considered as a possibility when young patients complain of atypical abdominal pain. It is a particularly important concern in emergency departments, where a prompt diagnosis and surgery can enable the preservation of the spleen.

Herein we present a case of WS without early diagnosis. The patient was referred to the emergency department with acute abdomen syndrome due to pedicle torsion. After examination, a splenectomy was performed. This paper was written to emphasize the importance of early diagnosis and treatment for this condition.

\section{REFERENCES}

1. Soleimani M, Mehrabi A, Kashfi A, Fonouni $\mathrm{H}$, Büchler MW, Kraus TW. Surgical treatment of patients with wandering spleen: report of six cases with a review of the literature. Surg Today. 2007;37(3):261-9.

2. DeJohn LM, Hal HM, Winner LS Wandering spleen: a rare diagnosis with variable presentation. Radiol Case Rep. 2008;3(4):1-6.

3. Awan M, Gallego JL, Al Hamadi A, Vinod VC. Torsion of wandering spleen treated by laparoscopic splenopexy: A case report. Int J Surg Case Rep. 2019;62:58-61.

4. Liu HTM, Lau KK. Wandering spleen: an unusual association with gastric volvulus. AJR Am J Roentgenol. 2007;188(4):W328-30.

5. Makhlouf NA, Morsy KH, Ammar S, Mohammed RA, Yousef HA, Mostafa MG. Wandering spleen in the pelvic region in an adult man with symptoms of acute abdomen. Arab J Gastroenterol. 2016;17(1):49-52.

6. Vaynshtein J, Guetta O, Replyansky I, Vakhrushev A, Czeiger D, Ovnat $A$, et al. Wandering spleen : three subsequent cases in young women. Isr Med Assoc J. 2018;20(10):656-7.
7. Yakan S, Telciler KE, Denecli AG. Acute torsion of a wandering spleen causing acute abdomen. Hong Kong J Emerg Med. 2011;18(1):34-6.

8. Kapan M, Gumuş M, Onder A, Gumuş $\mathrm{H}$, Aldemir M. A wandering spleen presenting as an acute abdomen: case report. J Emerg Med. 2012;43(5):e303-5.

9. Rafie BA, AbuHamdan OJ, Trengganu $\mathrm{NS}$, Althebyani BH, Almatrafi BS. Torsion of a wandering spleen as a cause of portal hypertension and mesenteric varices: a rare aetiology. J Surg Case Rep. 2018;5:1-4.

10. Varga I, Babala J, Kachlik D. Anatomic variations of the spleen: current state of terminology, classification, and embryological background. Surg Radiol Anat. 2018;40:21-9.

11. Karapolat B, Korkmaz HAA, Kocak G. Torsion of the wandering spleen. Am J Med Sci. 2019;357(5):e17-8.

12. Wallace S, Herer E, Kiraly J, Valikangas E, Rahmani R. A wandering spleen: unusual case of a pelvic mass. Obstet Gynecol. 2008;112(2):478-80.
13. Pagoti R, Young E, Gie CA, Moorby T. Ectopic spleen presenting as a pelvic mass. Int J Gynecol Obstet. 2007;96(1):51-2.

14. Sato M, Miyaki Y, Tochikubo J, Onoda T, Shiiya N, Wada H. Laparoscopic splenectomy for a wandering spleen complicating gastric varices: report of a case. Surg Case Rep. 2015;1(1):1-4.

15. Zarroug AE, Hashim Y, El-Youssef M, Zeidan MM, Moir CR. Wandering spleen as a cause of mesenteric and portal varices: a new etiology? J Pediatr Surg. 2013;48(3):E1-4.

16. Puranik AK, Mehra R, Chauhan S, Pandey R. Wandering spleen: a surgical enigma. Gastroenterol Rep. 2015;5(3):241-3.

17. Magowska A. Wandering spleen: a medical enigma, its natural history and rationalization. World J Surg. 2013;37(3):545-50.

18. Turhan AN, Kapan S, Gonenc M, Dogan M, Aygun E. Wandering spleen: report of two cases. Int Med Case Rep J. 2010;3:19-22. 